\title{
HEARTBEAT CLASSIFICATION USING THE RECURRENT NEURAL NETWORK BASED ON THE DEVELOPED SPIDER MONKEY-BIRD SWARM OPTIMIZATION ALGORITHM- PROPOSED METHOD
}

\author{
K Koteswara Rao ${ }^{1}$, G Lalitha Kumari ${ }^{2}$ and Y Surekha ${ }^{3}$ \\ ${ }^{123}$ Department of CSE, PVPSIT, Vijayawada, Andhra Pradesh, India \\ ${ }^{1}$ koteswara2003@yahoo.co.in
}

\begin{abstract}
Even though several methods are introduced to perform the heart beat classification by using the ECG signals, but classifying the heartbeat by analyzing the cardiac arrhythmia is still a challenging task. Hence, an effective RNN-based SM-BS optimization algorithm is introduced in this work, which is the combination of the SMO and the BSA algorithm. As the SMO and BSA are integrated together, the features from both the optimization will be utilized in the proposed work. As SMO uses the intelligent behavior and BSA mimics the foraging behavior, flight behavior, and vigilance behavior. Both these algorithms are effectively used to optimize the problem. Hence, this can be fused with the RNN to perform the beat classification in the proposed work.
\end{abstract}

Keywords-ECG, SMO, BSA, RNN

\section{INTRODUCTION AND ORIGIN OF THE RESEARCH PROPOSAL}

Electrocardiogram (ECG) is one of the inexpensive and non-persistent diagnostic tools used in most of the applications in the medical field [1]. ECG signal reflects the heart activity and is difficult to analyse, as it is a non-stationary signal [13]. The variations made in the electrical activity are represented based on the beats of the heart with respect to time and it contains the physiological information, which is used to analyze the function of the heart [1]. Each heartbeat are recorded by the ECG and from the recorded characteristic features; the subject class of the persons are identified [6]. The electrodes are used to record the electric field of cardiac in the body surface, which is the potential difference between the electrodes. The imaginary lines are formed in the body by combining the electrodes and the electric signals are considered as lead [1]. ECG contains six unipolar lead also referred as V leads or precordial and three bipolar leads. Each lead in the signals are viewed as an electrical activity in the body using the heartbeat and for each lead, the record nearly contains $2.5 \mathrm{~s}$ duration. The prolonged recording is used from the lead to offer the rhythm strip of $10 \mathrm{~s}$ for accurately accessing the cardiac rhythm [3]. The ECG signals are considered as the periodic signals, as they are sequence of waves which changes periodically over time. The QRS complex is the main characteristic features of the ECG signal, which is the combination of $\mathrm{Q}$ wave, $\mathrm{R}$ wave, and $\mathrm{S}$ wave respectively. The complex analysis of the signal is corresponds to the beat analysis [1].

Received: July 17, 2019

Reviewed: January 15, 2020

Accepted: January 20, 2020 
Cardiac arrhythmia is one of the classes of cardiovascular disease, which gradually affects the function of the heart or may cause sudden death [2]. Arrhythmia is associated with the heart disease and ECG is employed in the clinical routine by the cardiologist to classify the heartbeat [3]. Arrhythmia is a rhythm or rate of heartbeat, which is irregularity with respect to time. The heart can beat with irregular rhythm, too slow or too fast at the time of arrhythmia. During arrhythmia, enough blood are not pumped by the heart to the human body, these blood flow lack damages the heart, brain and other parts in the body [1]. Most of the cardiac arrhythmia diagnosis is based on the ECG [2]. Classifying the heartbeat is one of the most ECG analysis approach to detect the arrhythmias [5]. The cardiac arrhythmias are classified into two types, as non life threatening and life threatening arrhythmia. The life threatening arrhythmia, like tachycardia and ventricular fibrillation leads to sudden death by triggering the cardiac arrest. The non life threatening leads to heart failure, hence the heart function weakening is reduced by using the therapy [1]. Classifying the heartbeat from the ECG signals is a precious tool in studying the cardiac arrhythmia, but it is a challenging task in the biosignal analyzing field [7]. Different pattern recognition techniques are adopted to perform the classification and detection of arrhythmia and these techniques involves various steps to eliminate the artifacts and noise from the signal [9]. The cardiac arrhythmias classification based on the electrocardiographic is investigated in most of the research works, as they utilized three different aspects, such as classifiers, features, and evaluation mechanism [11].

Based on the features of the heart, the heart beats are classified and analyzing the ECG in an artificial manner is time consuming [2]. Hence, the researchers are intended to develop the automatic heartbeat classification techniques. Various automatic beat classification, involves waveform detection [15], heartbeat classification, baseline drift elimination [14], and feature extraction. In the core of analyzing the ECG automatically, beat classification is important in the research area. In the past years, the researchers proposed the classification methods, such as wavelet transforms [16] and waveform feature [17]. In the recent years, machine learning methods are introduced to enhance the efficiency in detecting the arrhythmia to record the beat classification. The machine learning approach includes Independent Component Analysis (ICA), Hermite coefficients, wavelet features, and spectral features are developed in the beat classification [5]. Automatic beat classification is worth useful, where the work scans are partitioned into subject based and class based classes [5]. Some of the classifiers widely used in the beat classification are Self Organization Map Neural Network (SOMNN) [18], Probabilistic Neural Network (PNN) [19], Multilayer Perceptron Neural Network (MLPNN) [2] [19], and Dynamic Bayesian network [2] [21], neural networks (NN), and support vector machines (SVMs) [9]. The current classification schemes are modeled based on the training of the sample base. Machine learning model requires the on-line learning ability to classify the patient heart beat based on their characteristics. By accumulating the medical data, automatic classification approach is developed by using the characteristic features of heartbeat of each person [2].

Even though several methods are introduced to perform the heart beat classification by using the ECG signals, but classifying the heart beat by analyzing the cardiac arrhythmia is still a challenging task. Hence, an effective RNN-based SM-BS optimization algorithm is introduced in this work, which is the combination of the SMO and the BSA algorithm. As the SMO and BSA are integrated together, the features from both the optimization will be utilized in the proposed work. As SMO uses the intelligent behavior and BSA mimics the foraging behavior, flight behavior, and vigilance behavior. Both these algorithms are effectively used to optimize the problem. Hence, this can be fused with the RNN to perform the beat classification in the proposed work. 


\section{LITERATURE SURVEY}

\subsection{INTERNATIONAL STATUS}

G. Sannino and G. De Pietro [1] developed a deep learning approach to achieve the hearbeats classification. It uses the tensor flow framework to detect the arrhythmia. The features encircled in the RR interval and the beat samples were utilized to classify the heartbeat. The abnormal beats are separated from the normal beats and attained good generalization ability. This approach is more efficient in terms of accuracy, but it achieved poor performance with respect to the specificity and sensitivity. Li P et al. [2] modeled a General Regression Neural Network (GRNN) to perform the heartbeat classification. The personalized classification scheme is constructed by using the GRNN for each patient. It saves the time of the doctors and minimizes the processing time. The performance of the GRNN is 450-fold increased using the GTX780Ti and attained significantly better sampling rate and efficiency. GRNN uses the multi computing cores based on the Graphics Processor Unit (GPU) to process the dataset parallely. In the GPU, the space in the memory is allocated and the computing tasks are performed. MondejarGuerraa $\mathrm{V}$ et al. [3] developed an electrocardiograms classification approach based on the Support Vector Machines (SVMs). SVM is used to train the feature types and the final prediction is obtained from the decision made using the sum, product, and majority rules. The normal and the abnormal beats are classified based on the features of the SVM model. It offers better results in the state of art features but it is failed to use the ensemble of SVM. The features obtained from each lead is separated and trained to combine the decision based on the product rule. Shi $\mathrm{H}$ et al. [4] modeled a hierarchical classification scheme based on the extreme gradient boosting (XGBoost). The heartbeats are preprocessed and the features are extracted from the processed beats. It uses the hierarchical classifier based on the XGBoost classifier and threshold to improve the weight. The XGBoost classifier divide the heartbeats into 'V-F' class and 'N-S' class and further the classification is employed into the classifier. However, the heartbeats collected and utilized in the rare class is limited. In the abnormal class, greater sensitivity and in the $\mathrm{N}$ class, greater predictive value is attained.

Chen S et al. [5] developed an electrocardiogram beat classification method to integrate the dynamic and the projected features to be an normalized form. In the projected features, the rows are converted by applying the Discrete Cosine Transform (DCT), whereas the columns are normalized. The dynamic features are determined by the weighted inter-beat (RR) interval and it utilizes the SVM classifier to cluster the beat using the features into one of five or 15 classes. In the subject based and class based strategy, this method attained better accuracy. However, the fiducial points were not detected from the heartbeat to perform the feature extraction and segmentation. ElSaadawy H et al. [6] introduced a two stage hierarchical approach for ECG classification. The heartbeat rate variability in the consequences were reduced by segmenting the heartbeats dynamically. The morphological features are extracted by using the wavelet transform. The features that were extracted is reduced and compressed by applying the principle component analysis. The RR features along with the obtained features are passed into the support vector machine classifier, which classifies the features into five different categories. Hence, the heartbeats are classified into the classes and the features are assigned to the classes based on their category. However, this method attained better accuracy, but failed to apply the stack generalization. Teijeiro $\mathrm{T}$ et al. [7] developed a knowledge based method to perform the beat classification automatically. The ECG signals are applied into the physiological process to extract the abstract features. For each beat, the rhythm and the qualitative morphological features were extracted by abducting the signal interpretation. The errors present in the signal interpretation is removed by the using the clustering algorithm. The clusters were generated and for each cluster a tag is 
assigned by the rule based classifier. The computing performance of this approach is improved, but the interpretation space is reduced. Ayar $M$ and Sabamoniri S [8] developed a hybrid approach to identify the features and classify the arrhythmias. It uses the genetic algorithm to identify the features optimally and the decition tree based scheme is applied into the features extracted to categorize and train this scheme. This scheme effectively classifies the data as abnormal or normal classes based on the type of arrhythmias. This scheme attained better sensitivity, accuracy and specificity in both two and 16 modes of class. However, it was not applicable for the dataset other than UCI arrhythmia.

Al Rahhal et al. [9] modeled a deep learning based approach for electrocardiogram signal classification. The features are represented in an unsupervised manner using the sparsity constraint based on the auto encoders of the stacked denoising from the data. At the hidden layer of representation, the regression layer using softmax is added to yield the neural network. The uncertain and the relevant beats are labeled in the records to update the weight of the neural network at each iteration. The expert interaction is minimized and the classification accuracy is increased and Suring the labeling process this method offers efficient computation. However, the deep learning was not combined with the content learning. Bousse $\mathrm{M}$ et al. [10] introduced a tensor-based method tensor based scheme to perform the irregular and single beat classification. The data matrix in ECG was tensorized to segment the heartbeat signal. The tensorized signal is resulted into the stacking based on the third order tensor using the beat dimensions. The resulted tensor was modeled by the multilinear, which depends on the singular value. Even though the performance was better, the channel was not determined in the classification. Afkhami $\mathrm{R}$ $\mathrm{G}$ et al. [11] introduced a beat classification method. The statistical and the morphological features were used in the classifier for the purpose of training. The RR features were used in the time-domain content, which acts as the role of exemplars. The density function of the beats was fitted by the expectation maximization approach. The decision trees and their ensemble were trained by the extracted statistical and the morphological features. The beat signals are processed by the mixture model and the classifiers were effectively trained. The classification of the beat signals was unevenly distributed in the arrhythmia class.

\subsection{NATIONAL STATUS}

Lakshminarayan $\mathrm{C}$ and Basil $\mathrm{T}$ [12] developed a machine learning approach to detect the heartbeat class. In the beat cycle, the features were extracted, which is further used to detect the class. However, it contains the time based and the morphological features with the dimensional vectors in an meaningful manner to detect the arrhythmia. The extracted features degraded the performance and the waves were not detected.

Celin S and Vasanth K [13] introduced the machine learning technique to investigate the heart function and classify the heartbeat. It uses the filtering process for the input signal to eliminate the noise with high frequencies. The excess noise was removed from the signal by using the butter worth filter. It utilizes the peak detection scheme to detect the peak points and the features were effectively extracted and classified using the naive bayes classifier. However, the accuracy of this scheme is better, but the performance is poor.

\section{IMPORTANCE OF THE PROPOSED RESEARCH}

The deep learning method in [1] classifies the normal and abnormal beats automatically. The precision was not evaluated using the available databases and the learning ability was not optimized. It was not embedded into the monitoring system in the real world environment. The heartbeat classification technique in [2] classifies the heartbeat using the medical instrumentation. However, analyzing the data is a tedious 
process and the time cost also increases. In [3], the SVM is used to classify the beat signals. Ensembling the SVM using the interval RR and the wavelet was not integrated with the product rule. The hierarchical classification scheme in [4] classifies the single heartbeat using the XGBoost classifier. It reduced the impact balance and the sensitivity was not obtained for the class. More hearbeats were not collected and used in the subject based classes. ECG beat classification method in [5] combines the dynamic and the projected features. The fiducial points were not detected and segmented and the signals were not analyzed in the compressed domain. Clustering algorithm in [7] uses the rhythm and the morphological features to reduce the interpretation errors. The beat labeling was not integrated in the test cycle and the real time constraints were not satisfied. The analysis of the existing methods of heart beat classification insists the researchers towards developing an effective method.

\section{BASIS OF THE INNOVATIVE ELEMENTS} field.

This section describes the importance of the heartbeat classification in the medical

- It is required to detect the rhythm abnormalities to measure the heart electrical activity and to separate the lead.

- To enhance the ECG recordings for diagnosing the no- life threatening arrhythmias as it aids a great worth studying in the detection of the signals.

- To enhance the quality of the features to perform further classification and beat detection mechanisms.

- To diagnose the cardiac arrhythmia effectively based on the ECG signals, and provides better detection rate for the transient ischemia, transient arrhythmia, and arrhythmia.

- To assure a healthy living scenario for the people through protecting them from mortality due to the cardiac problems

\section{PROPOSED METHODOLOGY AND PLANNING}

\subsection{METHODOLOGY}

Classification is the most important topic in the bioinformatics and the healthcare industry mainly for the purpose of arrhythmia detection. The primary aim of this work is to design and develop an effective heartbeat classification method based on SMBS- based RNN classifier. The proposed heartbeat classification method involves four stages, as preprocessing, heartbeat segmentation, feature extraction, and heartbeat classification. Initially, the input electrocardiogram (ECG) signal is passed into the signal preprocessing stage, where the baseline will be removed from the signal. The pre-processed signal will be subjected to the segmentation stage, which will use the QRS complex to perform the beat segmentation. The segmented result will be further passed into the feature extraction stage, where the features, like temporal features and wave features will be extracted. The temporal features, includes pre-RR interval, post-RR interval, local average RR interval, and global average interval. The wave features considered will be PP interval, PR interval, RR interval, QT interval, and R interval.

The significance of pre-processing the signals assists in the removal of the unwanted portions from the signal so that the extracted features are effective and accurate for heartrate classification. The extracted features will be forwarded to the classification stage, where the heartbeat classification will be performed using the proposed Spider MonkeyBird Swarm-based Recurrent neural network (SMBS- based RNN classifier). The proposed approach will use the combination of recurrent neural network and the Spider Monkey-Bird Swarm algorithm, which will be the integration of the Spider Monkey 
Optimization (SMO) and the Bird Swarm (BSA) algorithm. The SMO uses the foraging behavior of the spider monkeys in a fission-fusion like structure to solve the optimization problem, whereas the BSO is the generalization of the social interaction and the social behavior of the bird swarms. Moreover fusing the SMO with BSA efficiently extracts the foraging and the intelligent behavior of the bird swarms. The purpose of the optimization assures an effective tuning of RNN towards accurate prediction and classification. The proposed approach will be implemented in the MATLAB tool and the performance will be evaluated using the metrics, such as accuracy and good put.

Therefore the proposed approach effectively perform the heartbeat classification and will attain better accuracy and good put when compared with the existing techniques, as deep learning approach [1], hierarchical classification scheme [4], knowledge based method [7]. Figure 1 shows the block diagram of the proposed recurrent neural network based spider monkey-bird swarm optimization approach.

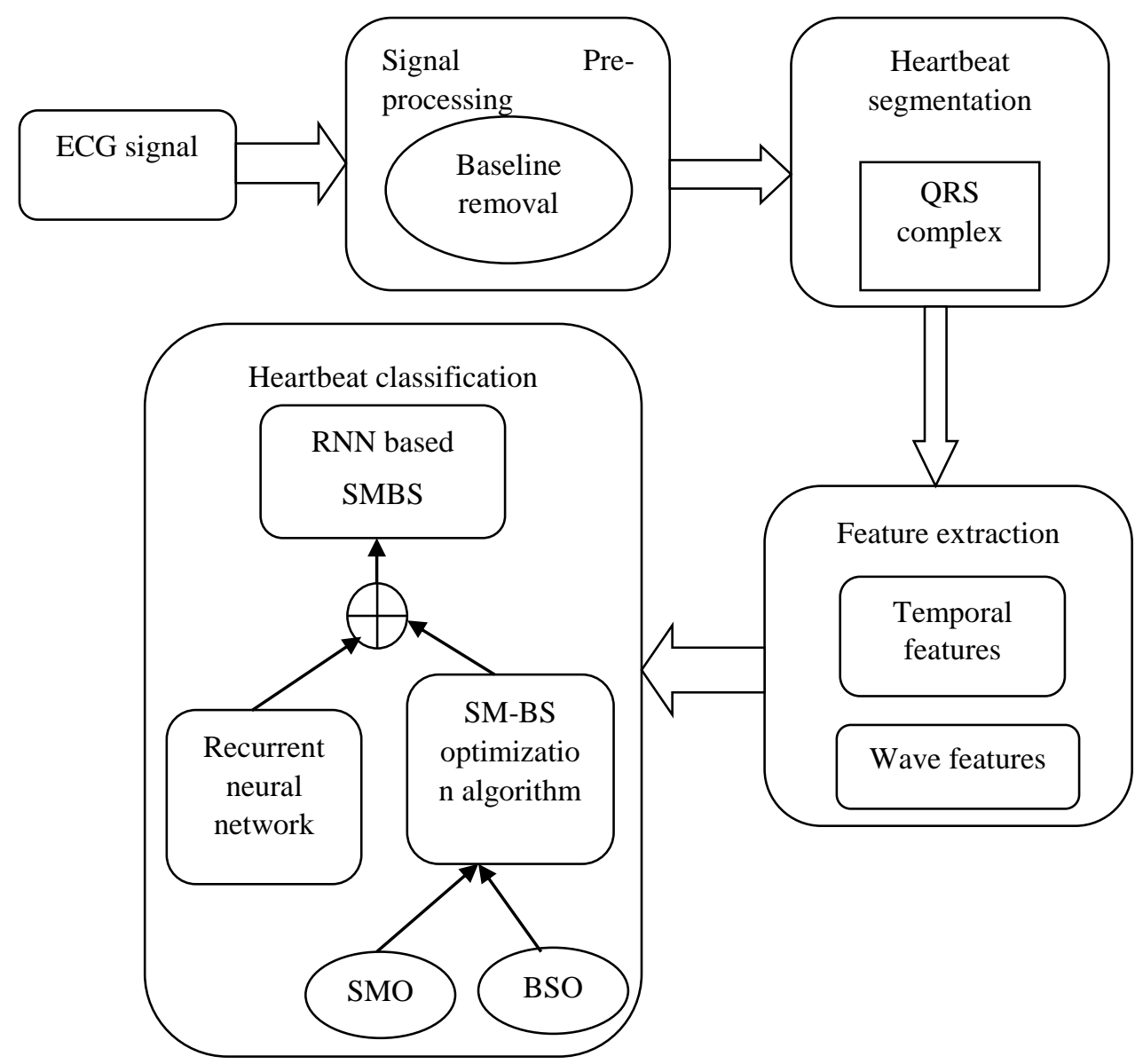

Fig. 1 Block diagram of the proposed recurrent neural network based spider monkeybird swarm optimization approach

\subsection{RESEARCH OUTCOMES EXPECTED FROM THE PROPOSED WORK}

The main objective of this work is to classify the heartbeat signal based on their characteristic features. Following are the suggested plan of action by utilizing the research outcome:

- Aim is to model an effective heartbeat classification by segmenting the preprocessed signals using the QRS complex.

- Goal is to classify the beat signal by using the recurrent neural network and the spider monkey bird swarm optimization algorithm in the extracted features. 
- Effectively performs the feature extraction and the resulted features, like temporal and the wave features are extracted.

- Good deliverable with enhanced features and can be used in the field of medical area by providing the optimal solution to the beat classification problem.

- It offers more beneficial for the doctors to classify and detect the cardiac arrhythmia based on the ECG.

\subsection{IDENTIFIED BENEFICIARIES}

The application of the heart beat classification benefits the normal persons all over the world, to have a deep insight over the health scenario of the individuals. Since the world is reporting a high risk for various deadly diseases related with the cardiac troubles, the early monitoring of the changes in the heart beat can assure the diagnosis in advance thereby, saving the life of humans. Moreover, the medical field is benefiting much as the time spend by the physician on a person is reduced and the life-style changes could be adapted to the rectify the abnormal reports in the heart beats.

\section{OBJECTIVES AND EXPECTED OUTCOMES}

- The main objective of the research is to develop a method for heart beat classification using ECG signals automatically.

- The accuracy of the classification is a critical factor to be concentrated for which the neural networks is employed.

- To remove the baseline present in the ECG signals and to generate the segmented image

- The temporal and the spatial features are extracted effectively to perform the classification process.

- To perform the heart beat classification effectively thereby, it attained better accuracy and good put.

In [1], it is reported that the performance of deep neural networks (DNN) is effective compared with the existing systems. However, the application of the ECG features enables the effective beat classification and decision-making using RNN-based SMBS optimization algorithm with the expected accuracy to be greater than 95\%.It is applicable in the real-world environment, especially in the medical field for the early detection of the heart diseases thus, insisting the researchers in the medical surroundings towards rendering an effective decision-making regarding the health scenario of the patients.

\section{REFERENCES}

[1] Sannino G and De Pietro G, "A deep learning approach for ECG-based heartbeat classification for arrhythmia detection”, Future Generation Computer Systems, vol. 86, pp.446-455, 2018.

[2] Li P, Wang Y, He J, Wang L, Tian Y, Zhou T.S, Li T and Li J.S, "High-performance personalized heartbeat classification model for long-term ECG signal", IEEE Transactions on Biomedical Engineering, vol. 64, no. 1, pp.78-86, 2017.

[3] Mondéjar-Guerra V., Novo J., Rouco J., Penedo M.G. and Ortega M., "Heartbeat classification fusing temporal and morphological information of ECGs via ensemble of classifiers", Biomedical Signal Processing and Control, vol. 47, pp.41-48, 2019.

[4] Shi H., Wang H., Huang Y., Zhao L., Qin C. and Liu C., "A Hierarchical Method Based on Weighted Extreme Gradient Boosting in ECG Heartbeat Classification", Computer Methods and Programs in Biomedicine, 2019.

[5] Chen S., Hua W., Li Z., Li J. and Gao X., "Heartbeat classification using projected and dynamic features of ECG signal", Biomedical Signal Processing and Control, vol. 31, pp.165-173, 2017.

[6] El-Saadawy H., Tantawi M., Shedeed H.A. and Tolba M.F., "Hybrid hierarchical method for electrocardiogram heartbeat classification”, IET Signal Processing, vol. 12, no. 4, pp.506-513, 2017. 
[7] Teijeiro T., Félix P., Presedo J. and Castro D, "Heartbeat classification using abstract features from the abductive interpretation of the ECG", IEEE journal of biomedical and health informatics, vol. 22, no. 2, pp.409-420, 2018.

[8] Ayar, M. and Sabamoniri, S, "An ECG-based feature selection and heartbeat classification model using a hybrid heuristic algorithm", Informatics in Medicine Unlocked, vol. 13, pp.167-175, 2018.

[9] Al Rahhal, M.M., Bazi, Y., AlHichri, H., Alajlan, N., Melgani, F. and Yager, R.R., "Deep learning approach for active classification of electrocardiogram signals", Information Sciences, vol. 345, pp.340354, 2016.

[10] Boussé, M., Goovaerts, G., Vervliet, N., Debals, O., Van Huffel, S. and De Lathauwer, L.," Irregular heartbeat classification using Kronecker product equations", In 39th Annual International Conference of the IEEE Engineering in Medicine and Biology Society (EMBC), pp. 438-441, July 2017.

[11] Afkhami, R.G., Azarnia, G. and Tinati, M.A., "Cardiac arrhythmia classification using statistical and mixture modeling features of ECG signals", Pattern Recognition Letters, vol. 70, pp.45-51, 2016.

[12] Lakshminarayan, C. and Basil, T., "Feature Extraction and Automated Classification of Heartbeats by Machine Learning", 2016.

[13] Celin, S. and Vasanth, K., "ECG Signal Classification Using Various Machine Learning Techniques", Journal of medical systems, vol. 42, no. 12, p.241, 2018.

[14] M. Milanesi, "Independent component analysis applied to the removal of motion artifacts from electrocardiographic signals," Med. Biol. Eng. Comput., vol. 46, no. 3, pp. 251-261, Mar. 2008.

[15] M. Merino, "Envelopment filter and K-means for the detection of QRS waveforms in electrocardiogram,” Medical Engineering \& Physics, vol. 37, no. 6, pp. 605-609, Jun, 2015.

[16] X. Jiang, "ECG arrhythmias recognition system based on independent component analysis feature extraction," in Proc. IEEE Region 10 Conf., pp. 1-4, Nov. 2006.

[17] L. Mariano and M. Juan Pablo, "Heartbeat classification using feature selection driven by database generalization criteria," IEEE Trans Biomed Eng, vol. 58, no. 3, pp. 616 - 625, 2011

[18] M. Lagerholm, "Clustering ECG complexes using Hermite functions and self-organizing maps," IEEE Trans. Biomed. Eng., vol. 47, no. 7, pp. 838-848, Jul. 2000.

[19] C. V. Banupriya and S. Karpagavalli, "Electrocardiogram Beat Classification using Probabilistic Neural Network, "Int. J. of Comput. Applicat. (IJCA), pp. 31-37, 2014. 